Mathematical Models and Methods in Applied Sciences

Vol. 27, No. 14 (2017) 2803-2807

(C) World Scientific Publishing Company

DOI: $10.1142 / \mathrm{S} 0218202517990019$

\title{
Author index Volume 27
}

Aceves-Sanchez, P. \& Mellet, A., Anomalous diffusion limit for a linear Boltzmann equation with external force field

Achdou, Y., Bardi, M. \& Cirant, M., Mean field games models of segregation

Alghamdi, M. A., see Gibelli

Althiabi, A. M., see Gibelli

Arcuri, A. \& Lanchier, N., Stochastic spatial model for the division of labor in social insects

Artina, M., Cagnetti, F., Fornasier, M. \& Solombrino, F., Linearly constrained evolutions of critical points and an application to cohesive fractures

Bardi, M., see Achdou

Barrenechea, G. R., John, V. \& Knobloch, P., An algebraic flux correction scheme satisfying the discrete maximum principle and linearity preservation on general meshes

Beirão da Veiga, L., Lovadina, C. \& Russo, A., Stability analysis for the virtual element method

Bellomo, N. \& Brezzi, F., Mathematical models of self-propelled particles

Bellomo, N. \& Ha, S.-Y., A quest toward a mathematical theory of the dynamics of swarms

Bellomo, N., Brezzi, F. \& Pulvirenti, M., Modeling behavioral social systems

Berrone, S. \& Borio, A., A residual a posteriori error estimate for the Virtual Element Method

Bertozzi, A. L., see Wang

Bitsouni, V., Chaplain, M.

27 (2017) $845 \quad$ A. J. \& Eftimie, R., Mathematical modelling of cancer invasion: The multiple roles of TGF- $\beta$ pathway on tumour proliferation and cell adhesion

Bobrowski, A., Kaźmierczak, B. \& Kunze, M., An averaging principle for fast diffusions in domains separated by semi-permeable membranes

Bonetti, E., Colli, P. \& Tomassetti, G., A nonsmooth regularization of a forward-backward parabolic equation

$\mathbf{2 7}(2017) 231$

27 (2017) 75

27 (2017) 525

27 (2017) 2557

27 (2017) 997

$27(2017) 745$

$27(2017) 1$

Bongini, M. \& Buttazzo, G., Optimal control problems in transport dynamics

Bongini, M., Fornasier, M., Hansen, M. \& Maggioni, M., Inferring interaction rules from observations of evolutive systems I: The variational approach

Borio, A., see Berrone

Bostan, M. \& Carrillo, J. A., Reduced fluid models for self-propelled particles interacting through alignment

Brezzi, F., see Bellomo

Brezzi, F., see Bellomo

Bru, J.-B. \& de Siqueira Pedra, W., Universal bounds for large determinants from noncommutative Hölder inequalities in fermionic

27 (2017) 1423

27 (2017) 205

27 (2017) 1929

27 (2017) 663

27 (2017) 641

27 (2017) 427

27 (2017) 909 27 (2017) 1423 constructive quantum field theory

27 (2017) 1963 
Buffa, A. \& Giannelli, C., Adaptive isogeometric methods with hierarchical splines: Optimality and convergence rates

Burini, D. \& Chouhad, N., Hilbert method toward a multiscale analysis from kinetic to macroscopic models for active particles

Buttazzo, G., see Bongini

Cáceres, E., Gatica, G. N. \& Sequeira, F. A., A mixed virtual element method for the Brinkman problem

Cagnetti, F., see Artina

Cai, Y., Shen, J. \& Xu, X., A stable scheme and its convergence analysis for a $2 \mathrm{D}$ dynamic $Q$-tensor model of nematic liquid crystals

Caponigro, M., Piccoli, B., Rossi, F. \& Trélat, E., Mean-field sparse Jurdjevic-Quinn control

Carles, R. \& Gallo, C., WKB analysis of generalized derivative nonlinear Schrödinger equations without hyperbolicity

Carrillo, J. A., see Bostan

Chaplain, M. A. J., see Bitsouni

Chechkin, G. A., Ratiu, T. S., Romanov, M. S. \& Samokhin, V. N., Existence and uniqueness theorems for the full threedimensional EricksenLeslie system

Chouhad, N., see Burini

Ciarlet, P. G. \& Iosifescu, O., Nonlinear Donati compatibility conditions on a surface - Application to the intrinsic approach for Koiter's model of a nonlinearly elastic shallow shell

Cirant, M., see Achdou

Colli, P., see Bonetti

Conti, S., Olbermann, H. \& Tobasco, I., Symmetry breaking in indented elastic cones

Crippa, G. \& Schulze, C., Cellular mixing with bounded palenstrophy
27 (2017) 2781

27 (2017) 1327

27 (2017) 427

27 (2017) 707

27 (2017) 231

27 (2017) 1459

27 (2017) 1223

27 (2017) 1727

27 (2017) 1255

27 (2017) 1929

27 (2017) 807

27 (2017) 1327

27 (2017) 347

27 (2017) 75

27 (2017) 641

27 (2017) 291

27 (2017) 2297
Crouseilles, N., Jin, S. \& Lemou, M., Nonlinear geometric optics methodbased multi-scale numerical schemes for a class of highly oscillatory transport equations

Degond, P., Frouvelle, A. \& Merino-Aceituno, S., A new flocking model through body attitude coordination

Degond, P., Liu, J.-G., Merino-Aceituno, S. \& Tardiveau, T., Continuum dynamics of the intention field under weakly cohesive social interaction

Della Corte, A., dell'Isola, F., Esposito, R. \& Pulvirenti, M., Equilibria of a clamped Euler beam (Elastica) with distributed load: Large deformations

dell'Isola, F., see Della Corte

de Siqueira Pedra, W., see Bru

Dick, J., Gantner, R. N., Le Gia, Q. T. \& Schwab, C., Multilevel higherorder quasi-Monte Carlo Bayesian estimation

Di Pietro, D. A. \& Droniou, J., $\quad W^{s, p}$-approximation properties of elliptic projectors on polynomial spaces, with application to the error analysis of a Hybrid High-Order discretisation of Leray-Lions problems

Djidjou-Demasse, R., Ducrot, A. \& Fabre, F., Steady state concentration for a phenotypic structured problem modeling the evolutionary epidemiology of spore producing pathogens

Droniou, J., see Di Pietro

Ducrot, A., see DjidjouDemasse

Eftimie, R., see Bitsouni

Ełaiw, A., see Gibelli

Elliott, C. M., Fritz, H. \& Hobbs, G., Small deformations of Helfrich energy minimising surfaces with
27 (2017) 953

27 (2017) 2031

27 (2017) 1005

27 (2017) 159

27 (2017) 1391

27 (2017) 1391

27 (2017) 1963

27 (2017) 879

27 (2017) 385

27 (2017) 879

27 (2017) 385

27 (2017) 1929

27 (2017) 617 
applications to biomembranes

Engwer, C., Stinner, C. \& Surulescu, C., On a structured multiscale model for acid-mediated tumor invasion: The effects of adhesion and proliferation

Esposito, R., see Della Corte

Evers, J. H. M., Fetecau, R. C. \& Sun, W., Small inertia regularization of an anisotropic aggregation model

Evje, S., Wen, H. \& Zhu, C., On global solutions to the viscous liquid-gas model with unconstrained transition to single-phase flow

Fabre, F., see DjidjouDemasse

Fetecau, R. C., see Evers

Fornasier, M., see Artina

Fornasier, M., see Bongini

Friedrich, M., A Korn-type inequality in SBD for functions with small jump sets

Fritz, H., see Elliott

Frouvelle, A., see Degond

Furioli, G., Pulvirenti, A., Terraneo, E. \& Toscani, G., Fokker-Planck equations in the modeling of socio-economic phenomena

Galam, S., Geometric vulnerability of democratic institutions against lobbying: A sociophysics approach

Gallo, C., see Carles

Gantner, G., Haberlik, D. \& Praetorius, D., Adaptive IGAFEM with optimal convergence rates: Hierarchical B-splines

Gantner, R. N., see Dick

Gao, Y. \& Li, P., Electromagnetic scattering for time-domain Maxwell's equations in an unbounded structure

Gatica, G. N., see Cáceres

Giannelli, C., see Buffa

Gibelli, L., Ełaiw, A., Alghamdi, M. A. \& Althiabi, A. M., Heterogeneous population dynamics of active particles: Progression, mutations, and selec-

27 (2017) 1795

27 (2017) 323

27 (2017) 385

27 (2017) 1795

27 (2017) 231

27 (2017) 909

27 (2017) 2461

27 (2017) 1547

27 (2017) 1005

27 (2017) 115

27 (2017) 13

27 (2017) 1727

27 (2017) 2631

27 (2017) 953

27 (2017) 1843

27 (2017) 707

27 (2017) 2781 tion dynamics

Giesselmann, J. \& Pryer, T., A posteriori analysis for dynamic model adaptation in convectiondominated problems

Giorgini, A., Grasselli, M. \& Miranville, A., The CahnHilliard-Oono equation with singular potential

Gong, S., see Wu

Grasselli, M., see Giorgini

Guo, Y. \& Liu, S., Incompressible hydrodynamic approximation with viscous heating to the Boltzmann equation

27 (2017) 617

27 (2017) 2381

27 (2017) 2485

27 (2017) 2711

27 (2017) 2485

27 (2017) 2261

Guo, Y. \& Wu, L., Regularity of Milne problem with geometric correction in $3 \mathrm{D}$

Ha, S.-Y., Ko, D. \& Zhang, Y., Critical coupling strength of the Cucker-Smale model for flocking

Ha, S.-Y., see Bellomo

Haberlik, D., see Gantner

Hansen, M., see Bongini

Henning, P. \& Peterseim, D., Crank-Nicolson Galerkin approximations to nonlinear Schrödinger equations with rough potentials

Hittmeir, S., Ranetbauer, H., Schmeiser, C. \& Wolfram, M.-T., Derivation and analysis of continuum models for crossing pedestrian traffic

Hobbs, G., see Elliott

Huang, B. \& Liao, Y., Global stability of combination of viscous contact wave with rarefaction wave for compressible Navier-Stokes equations with temperaturedependent viscosity

Huang, J. \& Shu, C.-W., A second-order asymptoticpreserving and positivitypreserving discontinuous Galerkin scheme for the Kerr-Debye model

Iosifescu, O., see Ciarlet
27 (2017) 549

27 (2017) 1051

27 (2017) 745

27 (2017) 2631

27 (2017) 909

27 (2017) 2147

27 (2017) 1301

27 (2017) 1547

27 (2017) 2321

27 (2017) 347 
Jerez-Hanckes, C., Schwab, C. \& Zech, J., Electromagnetic wave scattering by random surfaces: Shape holomorphy

Jin, S., see Crouseilles

John, V., see Barrenechea

Kanai, T., see Takizawa

Kaźmierczak, B., see Bobrowski

Klar, A., see Müller

Knees, D. \& Negri, M., Convergence of alternate minimization schemes for phase-field fracture and damage

Knobloch, P., see Barrenechea

Ko, D., see Ha

Krejčí, P., Rocca, E. \& Sprekels, J., Unsaturated deformable porous media flow with thermal phase transition

Kunze, M., see Bobrowski

Lachowicz, M., Leszczyński, H. \& Parisot, M., Blow-up and global existence for a kinetic equation of swarm formation

Lanchier, N., see Arcuri

Le Gia, Q. T., see Dick

Lemou, M., see Crouseilles

Leszczyński, H., see Lachowicz

Li, P., see Gao

Li, Y. \& Zhu, P., Asymptotics toward a nonlinear wave for an outflow problem of a model of viscous ions motion

Liao, Y., see Huang

Liu, J.-G., see Degond

Liu, S., see Guo

Lohéac, J., Trélat, E. \& Zuazua, E., Minimal controllability time for the heat equation under unilateral state or control constraints

Lovadina, C., see Beirão da Veiga

Maggioni, M., see Bongini

Martin, S., see Pinnau

Mellet, A., see AcevesSanchez

Merino-Aceituno, S., see Degond

Merino-Aceituno, S., see Degond

Meurer, A., see Müller
27 (2017) 2229

27 (2017) 2031

27 (2017) 525

27 (2017) 771

27 (2017) 663

27 (2017) 1177

27 (2017) 1743

27 (2017) 525

27 (2017) 1051

27 (2017) 2675

27 (2017) 663

27 (2017) 1153

27 (2017) 45

27 (2017) 953

27 (2017) 2031

27 (2017) 1153

27 (2017) 1843

27 (2017) 2111

27 (2017) 2321

27 (2017) 159

27 (2017) 2261

27 (2017) 1587

27 (2017) 2557

27 (2017) 909

27 (2017) 183

27 (2017) 845

27 (2017) 159

27 (2017) 1005

27 (2017) 1177
Michel, P., Large population and size scale limit of a stochastic particle model

Miranville, A., see Giorgini

Mirrahimi, S., A HamiltonJacobi approach to characterize the evolutionary equilibria in heterogeneous environments

Müller, L., Meurer, A., Schneider, F. \& Klar, A., A numerical investigation of flux-limited approximations for pedestrian dynamics

Nakamura, T. \& Nishibata, S., Existence and asymptotic stability of stationary waves for symmetric hyperbolic-parabolic systems in half-line

Negri, M., see Knees

Nishibata, S., see Nakamura

Olbermann, H., see Conti

Parisot, M., see Lachowicz

Patrizi, S. \& Valdinoci, E., Long-time behavior for crystal dislocation dynamics

Peterseim, D., see Henning

Piccoli, B., see Caponigro

Pinnau, R., Totzeck, C., Tse, O. \& Martin, S., A consensus-based model for global optimization and its mean-field limit

Poyato, D. \& Soler, J., Euler-type equations and commutators in singular and hyperbolic limits of kinetic Cucker-Smale models

Praetorius, D., see Gantner Pryer, T., see Giesselmann

Pulvirenti, A., see Furioli

Pulvirenti, M., see Bellomo

Pulvirenti, M., see Della Corte

Ranetbauer, H., see Hittmeir

Ratiu, T. S., see Chechkin Rindler, F., Schwarzacher, S. \& Süli, E., Regularity and approximation of strong solutions to rateindependent systems

Rocca, E., see Krejčí

Röger, M. \& Schweizer, B., Strain gradient viscoplasticity with dislocation
27 (2017) 581

27 (2017) 2485

27 (2017) 2425

27 (2017) 1177

27 (2017) 2071

27 (2017) 1743

27 (2017) 2071

27 (2017) 291

27 (2017) 1153

27 (2017) 2185

27 (2017) 2147

27 (2017) 1223

27 (2017) 183

27 (2017) 1089

27 (2017) 2631

27 (2017) 2381

27 (2017) 115

27 (2017) 1

27 (2017) 1391

27 (2017) 1301

27 (2017) 807

27 (2017) 2511

27 (2017) 2675 
densities contributing to the energy

Romanov, M. S., see Chechkin

Rossi, F., see Caponigro

Rossi, R. \& Thomas, M., From adhesive to brittle delamination in viscoelastodynamics

Russo, A., see Beirão da Veiga

Samokhin, V. N., see Chechkin

Schmeiser, C., see Hittmeir

Schmidt, B., A GriffithEuler-Bernoulli theory for thin brittle beams derived from nonlinear models in variational fracture mechanics

Schneider, F., see Müller

Schulze, C., see Crippa

Schwab, C., see Dick

Schwab, C., see JerezHanckes

Schwarzacher, S., see Rindler

Schweizer, B., see Röger

Sequeira, F. A., see Cáceres

Shen, J., see Cai

Shen, J., see Yang

Short, M. B., see Wang

Shu, C.-W., see Huang

Soler, J., see Poyato

Solombrino, F., see Artina

Sprekels, J., see Krejčí

Stinner, C., see Engwer

Süli, E., see Rindler

Sun, W., see Evers

Surulescu, C., see Engwer

Takizawa, K., Tezduyar, T. E. \& Kanai, T., Porosity models and computational methods for compressible-flow aerodynamics of parachutes with geometric porosity

Tan, C., A discontinuous Galerkin method on kinetic flocking models

Tang, H., see Wu

Tao, Y. \& Winkler, M., Effects of signaldependent motilities in a Keller-Segel-type reaction-diffusion system

Tardiveau, T., see Degond

Terraneo, E., see Furioli

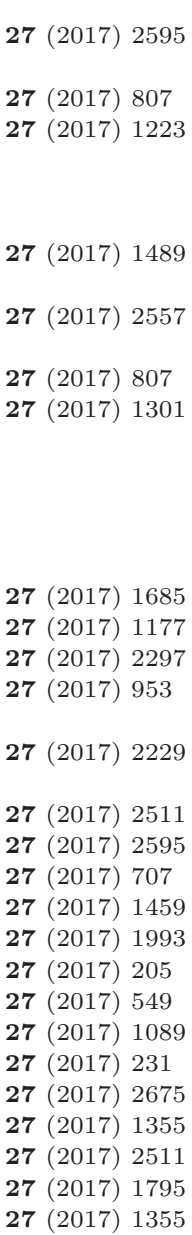

27 (2017) 771

27 (2017) 1199 27 (2017) 1871

27 (2017) 1645 27 (2017) 159 27 (2017) 115
Tezduyar, T. E., see Takizawa

Thomas, M., see Rossi

Tobasco, I., see Conti

Tomassetti, G., see Bonetti

Toscani, G., see Furioli

Totzeck, C., see Pinnau

Trélat, E., see Caponigro

Trélat, E., see Lohéac

Tse, O., see Pinnau

Valdinoci, E., see Patrizi

Wang, L., Short, M. B. \&

Bertozzi, A. L., Efficient

numerical methods for multiscale crowd dynamics with emotional contagion

Wang, Q., see Yang

Wang, Y., Global weak solutions in a threedimensional Keller-SegelNavier-Stokes system with subcritical sensitivity

Wen, H., see Evje

Winkler, M., see Tao

Wolfram, M.-T., see Hittmeir

Wu, K. \& Tang, H., Admissible states and physicalconstraints-preserving schemes for relativistic magnetohydrodynamic equations

Wu, L., see Guo

Wu, S., Gong, S. \& Xu, J., Interior penalty mixed finite element methods of any order in any dimension for linear elasticity with strongly symmetric stress tensor

$\mathrm{Xu}, \mathrm{J}$., see $\mathrm{Wu}$

$\mathrm{Xu}, \mathrm{X}$., see Cai

Yang, X., Zhao, J., Wang, Q. \& Shen, J., Numerical approximations for a three-component CahnHilliard phase-field model based on the invariant energy quadratization method

Zech, J., see Jerez-Hanckes

Zhang, Y., see Ha

Zhao, J., see Yang

Zhu, C., see Evje

Zhu, P., see Li

Zuazua, E., see Lohéac
27 (2017) 771

27 (2017) 1489

27 (2017) 291

27 (2017) 641

27 (2017) 115

27 (2017) 183

27 (2017) 1223

27 (2017) 1587

27 (2017) 183

27 (2017) 2185

27 (2017) 205

27 (2017) 1993

27 (2017) 2745

27 (2017) 323

27 (2017) 1645

27 (2017) 1301

27 (2017) 1871

27 (2017) 453

27 (2017) 2711

27 (2017) 2711

27 (2017) 1459

27 (2017) 1993

27 (2017) 2229

27 (2017) 1051

27 (2017) 1993

27 (2017) 323

27 (2017) 2111

27 (2017) 1587 